For reprint orders, please contact reprints@future-science.com

\title{
Detecting and monitoring NO, SNO and nitrite in vivo
}

The detection and quantification of nitric oxide and related reactive nitrogen species in vivo is vital to the understanding of the pathology and/or treatment of numerous conditions. To that end, several detection and quantification methods have been developed to study NO, as well as its redox relatives, nitrite and S-nitrosothiols. While no single technique can offer a complete picture of the nitrogen cycle in a given system in vivo, familiarity with the benefits and limitations of several common tools for $\mathrm{NO}_{x}$ determination can assist in the development of new diagnostics and therapeutics.

Keywords: chemiluminescence • electron paramagnetic resonance spectroscopy • spin trap

Nitric oxide (NO) is an important bioactive molecule with numerous roles in physiological systems and potential therapeutic applications, many of which are discussed throughout this issue. Understanding the role of $\mathrm{NO}$, particularly in cases where $\mathrm{NO}$ homeostasis may be disrupted or NO regulation is intrinsically tied to pathology, relies on the accurate detection and quantification of this molecule. Many techniques exist for both the direct and indirect quantification of $\mathrm{NO}$ and related nitrogen species (including nitrite/nitrate and $S$-nitrosothiols) (Table 1). Each method has its advantages under certain experimental conditions and with regard to specific targets, but no single method allows for complete, unrestricted quantification of $\mathrm{NO}$ in vivo (summarized in Table 2). However, depending on the desired test parameters, many techniques or combinations of techniques can be used to collect data on $\mathrm{NO}$ concentrations in physiological systems.

\section{Electrochemical probes}

Real-time, in vivo generation of $\mathrm{NO}$ can be detected by electrochemical sensors. Such probes utilize a system of two or three microelectrodes (a working electrode, a reference electrode and in some systems, an auxiliary electrode) to oxidize $\mathrm{NO}$ to $\mathrm{NO}^{+}$, which results in a small redox current (in the range of picoAmps to nanoAmps). By measuring this redox current in the system of interest and comparing it to the redox current between the electrodes when calibrated using NO standards, nearly real-time (requiring $30-60 \mathrm{~s}$ to equilibrate) $\mathrm{NO}$ concentrations can be monitored in vivo.

Working electrodes at a potential of 0.8-0.9 V relative to a silver/silver chloride reference electrode oxidize $\mathrm{NO}$ to $\mathrm{NO}^{+}$. Early electrochemical probes used working electrodes that were either gold-plated or made of pure platinum, though more recently, an alloy of platinum $(90 \%)$ and iridium (10\%) has been suggested due to its improved durability over pure Pt and the electrocatalytic properties of Ir in redox reactions [1]. Alternatively, carbon fiber working electrodes have been shown to have sensitivity comparable to gold-plated electrodes, and have the distinct advantage of being far smaller $(\sim 5 \mu \mathrm{m}$ diameter) than other electrodes (i.e., $\sim 130 \mu \mathrm{m}$ diameter for the Pt-Ir electrode) [2].

Care must be taken with working electrodes to prevent interaction with anions such as nitrite, nitrate, ascorbate or urates; pretreating the electrode with Nafion repels anions. Moreover, oxidation of nonnegative small molecules, such as dopamine and
Landon Bellavia ${ }^{1,2}$, Daniel B Kim-Shapiro*,1 \& S Bruce King ${ }^{3}$ 'Department of Physics, Wake Forest University, 1834 Wake Forest Rd, Winston-Salem, NC 27106, USA ${ }^{2}$ Department of Physical Science, The University of Findlay, 1000 N Main St, Findlay, OH 45840, USA ${ }^{3}$ Chemistry Department, Wake Forest University, 1834 Wake Forest Rd, Winston-Salem, NC 27106, USA *Author for correspondence: Tel.: +1 3367584993 shapiro@wfu.edu 


Table 1. Nitrogen species and their oxidation
states.
\begin{tabular}{ll} 
Compound, structure & Oxidation state \\
Nitrate, $\mathrm{NO}_{3}^{-}$ & +5 \\
Nitrogen dioxide, $\mathrm{NO}_{2}$ & +4 \\
\hline Nitrite, $\mathrm{NO}_{2}^{-}$ & +3 \\
\hline Nitric oxide, $\mathrm{NO}$ & +2 \\
\hline Nitroxyl, $\mathrm{HNO}$ & +1 \\
\hline Nitrogen, $\mathrm{N}_{2}$ & 0 \\
\hline Hydroxylamine, $\mathrm{NH}_{2} \mathrm{OH}$ & -1 \\
\hline Ammonia, $\mathrm{NH}_{3}$ & -3 \\
\hline
\end{tabular}

5-HT, can also result in overestimation of NO levels; this can be partially compensated for by pretreating electrodes in $o$-phenylenediamine to reduce interaction with cations [3].

Unfortunately, the extreme variability between different electrodes casts doubt on the utility of such devices for NO quantification. Different working electrodes can vary by as much as six orders of magnitude when attempting to quantify $\mathrm{NO}$ in vivo, despite extensive calibration against standards. Recent work suggests that this is due in part to electrode size, with larger electrodes underestimating $\mathrm{NO}$ due to rapid NO consumption from an unstirred layer; this work suggests that smaller electrodes are preferable not only for decreased invasiveness, but also for increased accuracy [4].

\section{Electron paramagnetic resonance}

Electron paramagnetic resonance (EPR) spectroscopy is a technique that can, in theory, detect the paramagnetic species in a sample (i.e., species that have one or more unpaired electrons, including free radicals such as NO). However, in practice, several factors may limit the applicability of EPR to any given system. $\mathrm{NO}$ is difficult or impossible to detect directly via EPR in most biological systems due to its broad, indistinct EPR signal in aqueous solution. However, it is possible to take advantage of the unique chemical properties of $\mathrm{NO}$ in order to create a reaction product, adduct or intermediate with a resolvable EPR spectrum.

Conventional diamagnetic spin trapping agents (e.g., nitrone-based traps), which are widely used to detect transient free radicals, are often not effective traps for NO. However, nitronyl nitroxides (NNOs), most notably carboxy-PTIO (c-PTIO), have been shown to be extremely effective at scavenging and trapping NO, resulting in a chemical shift that allows realtime EPR observation of the scavenging of NO. The efficacy of this assay is strongly dependent on the use of a proper amount of NNO for the experimental con- ditions. Moreover, both NNO reactants and products are easily reduced in many biological systems to EPR silent species, limiting their utility in quantification of NO production over time. NNOs can also directly interfere with NO generating pathways, or provide new reactive pathways for the generation of NO. These issues, coupled with the fact that NNOs (specifically c-PTIO) have recently been shown to react with other radicals, such as $\mathrm{NO}_{2}$, have led to a decrease in the use of NNOs to quantify $\mathrm{NO}$ generation in vivo via $\mathrm{EPR}$, except under specific conditions [5].

Other families of spin traps exist for the purpose of isolating NO, many of which utilize iron to bind $\mathrm{NO}$ and create an adduct that can be detected by EPR. The most common family of synthetic iron-based spin traps are iron-dithiocarbomate-based traps, including diethyldithiocarbamate [6] and $N$-methyl-D-glucamine dithiocarbamate (MGD) [5]. These spin traps have distinct EPR spectra at room temperature, allowing for real-time observation of the trapping of $\mathrm{NO}$, and can be used in conjunction with isotope substitution to identify sources of endogenous NO. However, they oxidize easily under aerobic conditions to create ferric complexes and reactive oxygen species, and may themselves generate $\mathrm{NO}$ from $\mathrm{HNO}$, nitrite or $S$-nitrosothiols.

Among the many biological targets for reaction with $\mathrm{NO}$, hemoglobin $(\mathrm{Hb})$ and myoglobin $(\mathrm{Mb})$ are some of the most relevant, and can also be used for the detection and quantification of NO. NO reacts or coordinates with ferrous heme depending on the presence or absence of oxygen. If the ferrous heme is oxygenated (forming oxyhemoglobin, oxyHb), NO reacts to form nitrate and ferric methemoglobin (metHb). MetHb at concentrations above approximately $0.5 \mu \mathrm{M}$ is easily resolved and quantified by EPR, even in a background with high concentrations of other $\mathrm{Hb}$ species, though the NO is consumed in its formation. Because oxyHb reacts very quickly with $\mathrm{NO}$ (with a rate constant between 5 and $8 \times 10^{7} \mathrm{M}^{-1} \mathrm{~s}^{-1}$ ), quantification of metHb formed from oxyHb during a process known to generate $\mathrm{NO}$ is often a reliable means of quantifying $\mathrm{NO}$ production [7]. If $\mathrm{NO}$ is present with a ferrous heme that is not coordinated with a ligand (deoxyhemoglobin, $\mathrm{Hb}$ ), the $\mathrm{NO}$ will coordinate with the $\mathrm{Hb}$ to form ferrous nitrosyl hemoglobin (HbNO). HbNO is relatively stable (with a dissociation rate on the order of $10^{-3}-10^{-4} \mathrm{~s}^{-1}$ ), and has a distinct EPR signal that can be used to quantify hemoglobin-bound NO at concentrations of approximately $0.5 \mu \mathrm{M}$ and above [8]. Additionally, because the $\mathrm{NO}$ is detected directly, isotope substitution can be used to trace the source of NO. Use of heme proteins in conjunction with EPR is limited by the fact that heme EPR spectra must be collected at 


\begin{tabular}{|c|c|c|}
\hline NO techniques & Benefits & Limitations \\
\hline \multirow[t]{3}{*}{ Electrochemical probes } & In situ data & Up to $10^{6}$ difference between techniques \\
\hline & \multirow[t]{2}{*}{ Nearly real time } & Electrode fragility and fouling \\
\hline & & Electrode pretreatment requirements \\
\hline \multirow[t]{2}{*}{ EPR: NNOs } & Highly effective NO trap & Nonspecificity \\
\hline & $\begin{array}{l}\text { Distinct spectral shift between } \\
\text { NO-bound and unbound states }\end{array}$ & $\begin{array}{l}\text { Reduced to EPR silent species under some } \\
\text { biological conditions }\end{array}$ \\
\hline \multirow[t]{3}{*}{ EPR: iron-dithiocarbomate spin traps } & Room temperature & Prone to oxidation \\
\hline & Real-time observation of NO trapping & Best under anaerobic conditions \\
\hline & Isotope substitution compatible & $\begin{array}{l}\text { May generate (and artificially detect) NO } \\
\text { from HNO, nitrite, S-nitrosothiols }\end{array}$ \\
\hline \multirow[t]{4}{*}{ EPR: metHb } & \multirow{2}{*}{$\begin{array}{l}\text { Quantifiable, with relatively low } \\
(500 \mathrm{nM}) \text { limit of detection }\end{array}$} & Aerobic conditions required \\
\hline & & Cryogenic temperatures $(\sim 5 K)$ \\
\hline & \multirow{2}{*}{$\begin{array}{l}\text { Detectable amid high concentrations of } \\
\text { hemes and free radicals }\end{array}$} & NO irreversibly consumed in reaction \\
\hline & & Does not directly detect NO \\
\hline \multirow[t]{2}{*}{ EPR: HbNO } & NO trapped & Partially oxygenated conditions only \\
\hline & Isotope substitution compatible & May detect nitrite as well \\
\hline \multirow[t]{3}{*}{ Fluorescence: DAFCs } & $\mathrm{NO}\left(\right.$ or $\mathrm{N}_{2} \mathrm{O}_{3}$ ) imaging in cells & Aerobic conditions required \\
\hline & Sensitivity: $5 \mathrm{nM}$ detection limit & $\begin{array}{l}\text { Reacts with oxidized NO metabolites, } \\
\text { especially } \mathrm{N}_{2} \mathrm{O}_{3}\end{array}$ \\
\hline & & Generates $\mathrm{N}_{2} \mathrm{O}_{3}$ intermediate \\
\hline \multirow[t]{3}{*}{ Fluorescence: copper fluoresceins } & Direct NO imaging in cells & Suboptimal emission wavelength \\
\hline & No $\mathrm{N}_{2} \mathrm{O}_{3}$ intermediate & Cytotoxicity \\
\hline & Sensitivity: $5 \mathrm{nM}$ detection limit & Potential instability in biological systems \\
\hline \multirow[t]{2}{*}{ Fluorescence: FRET } & Reversible & \multirow{2}{*}{$\begin{array}{l}\text { Detection limit of } 10 \mu \mathrm{M} \text { for } \\
\text { nongenetically modified cells }\end{array}$} \\
\hline & $\begin{array}{l}\text { NO detection limit of } 0.1 \mathrm{nM} \text { in } \\
\text { genetically modified cells }\end{array}$ & \\
\hline
\end{tabular}

DAFC: Diamino-aromatic fluorescent compound; EPR: Electron paramagnetic resonance; FRET: Fluorescence resonance enery transfer; HbNO: Nitrosyl hemoglobin; metHb: Methemoglobin.

cryogenic temperatures, and therefore measurements cannot be taken in real time or directly in vivo. In addition, EPR can only detect these reaction products at concentrations of a few hundred nanomolar or more.

\section{Fluorescence}

Beyond detection and quantification of $\mathrm{NO}$ in biological systems, direct imaging of $\mathrm{NO}$ distribution within systems promises even further insight into the role of this molecule in vivo. While several techniques have been developed for the fluorescent detection of NO, many of them have relatively poor detection thresholds and low specificity. Thus, three techniques are most commonly used for the direct imaging of $\mathrm{NO}$ in vivo [9].

The first successful fluorescent imaging of $\mathrm{NO}$ in vivo was carried out with diamino-aromatic fluorescent compounds (DAFCs). DAFCs (including DAF,
DAF-2, DAR, DAQ, DAF-FM-DA and others) react quantitatively with $\mathrm{NO}$ under aerobic physiological conditions to create fluorescent products with absorption and emission spectra in the visible or near-IR range, allowing for detection and imaging of $\mathrm{NO}$ concentrations above $5 \mathrm{nM}$. DAFCs do not react with nitrite and nitrate, allowing for $\mathrm{NO}$ imaging amid a background of nitrogen oxides at much higher concentrations than $\mathrm{NO}$ [9], nor do they react with peroxide, superoxide or peroxynitrite [10]. However each member of this family has unique advantages and drawbacks, such as $\mathrm{pH}$ sensitivity and specificity. The DAFC class of $\mathrm{NO}$ detectors is universally limited by the fact that they require oxygen in order to react with NO, limiting utility in hypoxic conditions, as well as by their propensity to react with oxidized NO products (notably $\mathrm{N}_{2} \mathrm{O}_{3}$ ) and other nitrogen-containing radicals and reducing agents [9]. 
To overcome many limitations of the DAFCs, a new family of copper (II) based fluorescent probes with sensitivity similar to DAFCs $(5 \mathrm{nM})$ was developed [11] and extensively explored (reviewed in [9]). These copper-based fluorescein derivatives react directly with NO, regardless of local oxygen concentration, and are specific to NO, ignoring other reactive nitrogen species, reactive oxygen species and ascorbate. Cell membranes are generally permeable to these copper-fluorescein molecules, allowing for intercellular and intracellular imaging of $\mathrm{NO}$ concentrations. However, they are limited by a suboptimal emission wavelength, as well as potential cytotoxicity and instability in vivo. Thus, use of these fluorescent probes has been largely limited to cell cultures, with benzimidazole derivatives having been used in frozen tissue samples.

DAFCs and copper-fluoresceins react with NO irreversibly, and may lead to the buildup of a high level of background fluorescence. To create a reversible NO detector, a fluorescent protein with the NOspecific heme domain of soluble guanylate cyclase was created. While both NO-specific and reversible, the threshold of detection for this molecule was only $10 \mu \mathrm{M}$. A related protein system with a much better detection limit $(0.1 \mathrm{nM})$ has been developed for use with FRET imaging, but this requires genetic encoding of the detector into the target organism. Future work in this area may lead to a more ubiquitous fluorescent NO detector, but current sGC- and FRET-related detectors, while they have a few unique advantages, are generally less useful than current DAFCs and copper-fluorescein fluorescent probes [9].

\section{Nitrite}

Nitric oxide generally has a relatively short half-life in vivo, ranging anywhere from tens of seconds in some tissues to milliseconds or microseconds in the presence of heme-containing proteins such as hemoglobin. With the correspondingly low steady-state concentration of $\mathrm{NO}$, real-time quantification of $\mathrm{NO}$ is difficult and at often times, the concentration of the nitrite and nitrate metabolites of $\mathrm{NO}$ will serve just as well as (or better than) the concentrations of NO itself [12]. Moreover, recent advances in NO chemistry have revealed that nitrite constitutes a significant reserve of bioavailable nitric oxide. This nitrite can be reduced to $\mathrm{NO}$ under hypoxic conditions by a variety of physiological processes, including acidic reduction and the reaction of nitrite with xanthine oxidoreductase, thiol-containing enzymes and deoxygenated heme proteins such as hemoglobin, myoglobin and neuroglobin. The ability of nitrite to rapidly form NO during hypoxic stress makes it a critical hypoxic buffer responsible for triggering vasodilation as oxygen levels drop [13]. Due to the intrinsic link between nitrite and $\mathrm{NO}$, accurate quantification of nitrite is important to understand systems of hypoxic vasodilation, hypoxic mitochondrial respiration and ischemia-reperfusion and to the development of nitrite-based treatments for conditions characterized by ischemia and hemodynamic dysregulation. To this end, several techniques have been developed or adopted for the quantification of nitrite in biological systems (summarized in Table 3).

\section{Griess reaction}

For over a century, the Griess reaction and its derivatives have been used to quantify nitrite in a variety of situations. However, application of the Griess reaction for in vivo quantification of nitrite presents unique challenges. For instance, the typical Griess assay has a detection limit of 1-2 $\mu \mathrm{M}$, a full order of magnitude above basal nitrite levels in many biological fluids such as plasma. Moreover, the reaction cannot be readily utilized in whole blood due to interference from blood constituents such as hemoglobin and plasma proteins. Thus, various techniques have been developed to improve sensitivity and facilitate blood, plasma and serum sample analysis using techniques based on the Griess reaction, each with advantages and disadvantages. The presence of even trace contaminants, anticoagulants and proteins can all reduce the accuracy of Griess-based assays [14,15].

Numerous sample preparation and storage methods have been used in the analysis of nitrite and nitrate concentrations by the Griess reaction, and some of the procedures used are not clear. Thus, even seemingly similar protocols can differ by an order of magnitude or more in their reported concentrations. Out of the commercially available storage monovettes, serum has been shown to have the lowest levels of nitrite contamination. EDTA and citrate, and to a lesser extent heparin, have been found to have significant levels of nitrite contamination, often greater than the nitrite concentration in the sample itself. Beyond selection of storage media, samples must be properly preserved and prepared for analysis by techniques based on Griess reactions. Nitrite can oxidize forming nitrate, a process that can be inhibited by alkali. Alkali is often combined with $\mathrm{ZnSO}_{4}$ to both prevent oxidation of nitrite and precipitate proteins from the solution that could potentially interfere with nitrite detection via nitrosation of cysteine or nitration/nitrosation of carbon or nitrogen under the acidic conditions necessary to conduct the Griess reaction [14]. Dilution of plasma samples in ethanol has also been shown to reduce the effect of 
Table 3. Benefits and limitations of select techniques for determination of nitrite.

\begin{tabular}{|lll|}
\hline $\begin{array}{l}\text { Nitrite techniques } \\
\text { Griess }\end{array}$ & $\begin{array}{l}\text { Benefits } \\
\text { Quantifiable reaction with nitrite } \\
\text { Provides a basis for modified assays }\end{array}$ & $\begin{array}{l}\text { Limitations } \\
\text { High limit of detection }(1-2 \mu \mathrm{M}) \text { in } \\
\text { assays using only Griess methods } \\
\text { Susceptible to contamination }\end{array}$ \\
\hline Chromatography & $\begin{array}{l}\text { Can be used in conjunction with Griess } \\
\text { technique, fluorochromes and others } \\
\text { Improves sensitivity up to } 500 \times\end{array}$ & $\begin{array}{l}\text { More difficult to standardize } \\
\text { Variation between instruments }\end{array}$ \\
& $\begin{array}{l}\text { Eliminates some pretreatment needs } \\
\text { Does not require deproteination } \\
\text { Very high sensitivity }(\sim 1 \mathrm{nM})\end{array}$ & $\begin{array}{l}\text { Specificity (potentially detects other } \\
\text { NO }\end{array}$ \\
& & Frequent calibration required \\
& & Variation between instruments \\
\hline
\end{tabular}

plasma proteins on the modified Griess reagents [15]. Due to the importance of deproteination to the success of the Griess assay, additional ultrafiltration or microdialysis techniques may be employed to purify samples, and many deproteination systems are built in to automated Griess assays that also involve other methods, such as chromatography [14].

\section{Chromatography}

Although the Griess reaction is the most widely cited technique for the quantification of nitrite, it is by no means the only method available, nor does it exclude the concurrent use of other techniques to improve the quality of data collection, detection limits and range of conditions under which data can be gathered. Numerous chromatography-based assays exist for the detection of nitrite (and often nitrate), frequently employing pre- or postcolumn treatments (sometimes based on the Griess reaction) to quantify these anions without interference from contaminants in the initial solution [12].

HPLC techniques can be used to quantify nitrite in conjunction with the Griess reaction described above or other methods of nitrite detection, such as the DAN fluorochrome (2,3-diaminonaphthalene) described elsewhere [16]. HPLC systems using either anion-exchange or polystyrene polymer columns have improved on quantification methods relying solely on the Griess reaction, improving sensitivity up to 500 -fold [14]. However, the results from such procedures are harder to standardize and can vary between instruments, requiring that great care be taken to validate each use.

Specialized systems designed and marketed for the detection of nitrogen oxides (typically nitrite and nitrate, collectively referred to as $\mathrm{NO}_{\mathrm{x}}$ ) employ both HPLC and spectrophotometric analysis of the diazo product of the Griess reaction. These specialized sys- tems have the advantage of highly reproducible results that can be obtained with less time-consuming sample preparation procedures. However, as with any commercial assay system, care must be taken to validate the assay in each new situation and to ensure consistent quality of results [12].

In addition to HPLC techniques, assays based on GC-MS have also been developed, with pentafluorobenzyl bromide (PFB-Br) used for the simultaneous derivatization and quantization of nitrite $[12,17]$. Recent work has used these techniques to quantify nitrite in whole blood rather than plasma or serum [17].

\section{Chemiluminescence}

Ozone-based chemiluminescence is a technique for the detection of NO gas that has been used with great success in a variety of applications. It is particularly useful in the determination of in vivo $\mathrm{NO}$ metabolites such as nitrite and nitrate, and can be used in situations where traditional Griess reaction assays and chromatography fail. This typically requires a specialized chemiluminescent detector, and such detectors designed for the quantification of $\mathrm{NO}$ are commercially available and well validated [18].

While the potential applications of chemiluminescence are numerous, it is a particularly valuable tool for the determination of nitrite and nitrate (as well as $S$-nitrosothiols, as discussed later in this article). Notably, chemiluminescence does not require deproteination prior to analysis, and can be conducted in the presence of hemoglobin and other contaminants, although oxidizing hemoglobin before analysis (e.g., through the use of ferricyanide) has been shown to be useful in the prevention of oxidation of nitrite to nitrate [19].

Chemiluminescent determination of nitrite or nitrate requires the reduction of the relevant $\mathrm{NO}_{\mathrm{x}}$ to $\mathrm{NO}$ within an anaerobic reaction vessel. Numerous 
reducing agents can be used, and different agents will reduce different $\mathrm{NO}_{\mathrm{x}}$ species; for example, tri-iodide $\left(\mathrm{I}_{3}{ }^{-}\right)$acidified with acetic acid can be used to reduce the majority of relevant $\mathrm{NO}_{x}$ species and is preferred for analysis of nitrite concentration (in addition to using potassium iodide alone), whereas copper(I)/cysteine is often used for the determination of $S$-nitrosothiols (discussed later in this issue).

Chemiluminescence is highly sensitive, with a detection threshold on the order of 1 pmol of total nitrite or $1 \mathrm{nM}$ of nitrite in solution, depending on the quantity of sample used. However, it also has limitations and potential pitfalls. Foaming (especially when using copper(I)/cysteine as a reductant) can cause an overflow from the reaction vessel when biological samples (particularly those with high protein levels) are injected into the reaction chamber. There is also a great deal of variability between instruments, and even on the same instrument between uses; thus, care must be taken to calibrate the instruments against a set of known standards before each use [18].

\section{S-nitrosothiols}

In addition to existing freely in solution or being oxidized to another species entirely, $\mathrm{NO}$ occurs in vivo as a protein modification factor. $\mathrm{NO}$ in the presence of oxygen can form $S$-nitrosothiols $(\mathrm{R}-\mathrm{S}-\mathrm{N}=\mathrm{O}$ ) (RSNOs). These RSNOs may transport NO activity, and also likely affect cellular NO signaling pathways. Thus, determination of the in vivo concentration of these RSNOs is important for understanding NO transport and signaling within the body. Quantification of small-molecule RSNOs at relatively high concentration in vitro can often be accomplished using UV/Vis spectroscopy, but the relatively low concentrations of these compounds in vivo, coupled with the fact that $S$-nitrosothiols of interest are often associated with large macromolecules, render spectroscopic techniques untenable. Thus, the most commonly used techniques for quantification of RSNOs generated in vivo rely on detection of liberated NO or the labeling of thiols vacated following the liberation of NO (summarized in Table 4).

\section{Chemiluminescence}

As described earlier, chemiluminescence can be used to detect the liberation of $\mathrm{NO}$ due to the reduction of nitrogen oxides. Chemiluminescent detection has been used to quantify $\mathrm{NO}$ release from the reduction of $S$-nitrosated proteins formed in vivo. Three reducing systems currently employed to facilitate this chemiluminescent detection assay are summarized here, and have been previously reviewed in greater detail [20].
In the tri-iodide $\left(\mathrm{I}_{3}^{-}\right)$method, potassium iodide (KI) and iodine $\left(\mathrm{I}_{2}\right)$ create tri-iodide in the presence of acetic acid. This serves as the reducing agent, simultaneously liberating NO from RSNOs, nitrosamines, iron-nitrosyl complexes and nitrite. Because of the less-specific nature of this assay, and the frequent contamination of materials by nitrite, care must be taken to maintain accurate controls [18].

Another system uses copper chloride, cysteine and carbon monoxide (the so-called 3C method). In this technique, $\mathrm{Cu}(\mathrm{I})$ reduces the nitrosothiol, while cysteine both reduces $\mathrm{Cu}(\mathrm{II})$ and forms CSNO. CO gas flow prevents any heme present from capturing $\mathrm{NO}$ before it can be detected. This method has the significant advantage of being nitrite silent, and thus ignores many otherwise-troublesome sources of contamination [21]. The 3C method is also convenient in that it requires no pretreatment of samples, though the difficulties caused by the use of CO may offset this advantage. CO, beyond the obvious health risks, can cause instrumental complications, such as overheating in some models of chemiluminescent detectors (notably in the hopcolite filter of the Sievers Nitric Oxide Analyzer, NOA 280i, which can be compensated for by placing ice packs around the filter). Protein-containing samples can also cause excessive foaming, requiring frequent replacement of the purge vessel solution and often making this procedure more time consuming than a comparable tri-iodide assay [20].

To overcome some of the difficulties with the 3C assay, a modified copper/cysteine procedure has been developed, frequently referred to as the modified $2 \mathrm{C}$ method. This uses pretreatment with $\mathrm{K}_{3} \mathrm{Fe}(\mathrm{CN})_{6}$, NEM and DTPA. $\mathrm{K}_{3} \mathrm{Fe}(\mathrm{CN})_{6}$ (ferricyanide) oxidizes hemoglobin to the non-NO capturing metHb state, while NEM blocks free thiols from becoming $S$-nitrosated during the treatment, and the metal chelator DTPA protects SNO bonds from free metals. The modified 2C method eliminates the complications of $\mathrm{CO}$ flow during the experiment, while preserving many of the 3C method's advantages (particularly nitrite independence). However, this method requires pretreatment of samples and additional care when dealing with some hemoglobin-based samples [20].

Each of these methods has advantages and disadvantages, but all three share certain key features. Perhaps most notably, while chemiluminescence can allow for accurate quantification of $\mathrm{NO}$ liberated from $S$-nitrosothiol reduction, it does not provide any insight into the specific location of that NO. For this, other techniques must be used, and can often be used in tandem with chemiluminescence for improved results. 
a coumarin-based phosphine was recently developed that fluoresces upon reaction of the phosphine with RSNO [25]. This molecule promisingly shows no reactivity with disulfides, although its general specificity for RSNOs has not yet been documented. It may also lack the necessary level of sensitivity, having only provided quantitative measurements of RSNO in the low $\mathrm{mM}$ range in vitro. However, the potential of such molecules, including the family of triarylphosphines [23], to serve as RSNO probes, coupled with the addition of fluorescent indicators such as this coumarin- phosphine, suggests that direct fluorescent detection of RSNOs in vivo may be attainable [24]. A recent report describes a mass spectrometric method for detecting small molecule $S$-nitrosothiols based on this phosphine chemistry [26].

\section{Conclusion \& future perspective}

The accurate detection and monitoring of NO, nitrite and $S$-nitrosothiols in vivo present numerous challenges, which depend largely on the conditions of study and the inherent difficulty in tracking transient species with short lifespans and relatively low physiological concentrations. While by no means an exhaustive list, many of the most useful and most widely used techniques, as well as some promising candidates for future investigation, have been summarized here. The interested researcher is encouraged to seek more details on any methods of interest in the articles referenced here. In the future improvements in fluorescence-based or mass-spectrometry-based techniques may improve in vivo detection. For now, there are many available techniques all of which can provide useful information provided proper controls are incorporated.

\section{Financial \& competing interests disclosure}

DB Kim-Shapiro is a principal investigator on NIH grants HL058091and HL098032 related to nitric oxide, nitrite and nitrate and this work was partially supported by these grants. The authors have no other relevant affiliations or financial involvement with any organization or entity with a financial interest in or financial conflict with the subject matter or materials discussed in the manuscript apart from those disclosed.

No writing assistance was utilized in the production of this manuscript.

\section{Open access}

This work is licensed under the Creative Commons Attribution 4.0 License. To view a copy of this license, visit http://www. creativecommons.org/licenses/by/4.0/

Executive summary

- There are many ways to investigate nitric oxide, nitrite and S-nitrosothiols in vivo, but none of them are universally applicable in all situations.

- NO can be determined by direct in vivo use of electrochemical probes (though these are subject to many limitations), electron paramagnetic resonance spectroscopy and fluorescence imaging.

- Nitrite can be determined by use of the Griess reaction and its derivatives, chromatography and chemiluminescence.

- S-nitrosothiols can also be quantified by chemiluminescence; determination of their location on proteins requires tagging the sites of $S$-nitrosation, such as by the Biotin switch technique or a derivative thereof.

\section{References}

1 Dalbasti T, Kilinc E. Microelectrode for in vivo real-time detection of NO. In: Methods in Enzymology. Lester P, Enrique C (Eds). Academic Press, CA, USA, 584-592 (2005).

2 Tsai AG, Acero C, Nance PR et al. Elevated plasma viscosity in extreme hemodilution increases perivascular nitric oxide concentration and microvascular perfusion. Am. J. Physiol. Heart Circul. Physiol. 288(4), H1730-H1739 (2005).

3 Hall CN, Garthwaite J. What is the real physiological NO concentration in vivo? Nitric Oxide 21(2), 92-103 (2009).

4 Bohlen HG. Is the real in vivo nitric oxide concentration pico or nano molar? Influence of electrode size on unstirred layers and NO consumption. Microcirculation 20 (1), 30-41 (2013).

5 Hogg N. Detection of nitric oxide by electron paramagnetic resonance spectroscopy. Free Rad. Biol. Med. 49(2), 122-129 (2010).
6 Cao Y, Guo P, Xu Y, Zhao B. Simultaneous detection of $\mathrm{NO}$ and ROS by ESR in biological systems. In: Methods in Enzymology, Lester P, Enrique C (Eds). Academic Press, CA, USA, 77-83 (2005).

7 Herold S, Exner M, Nauser T. Kinetic and mechanistic studies of the NO center dot-mediated oxidation of oxymyoglobin and oxyhemoglobin. Biochemistry-US 40(11), 3385-3395 (2001).

8 Azizi F, Kielbasa JE, Adeyiga AM et al. Rates of nitric oxide dissociation from hemoglobin. Free Rad. Biol. Med. 39(2), 145-151 (2005).

9 Hong H, Sun J, Cai W. Multimodality imaging of nitric oxide and nitric oxide synthases. Free Rad. Biol. Med. 47(6), 684-698 (2009).

10 Miller EW, Chang CJ. Fluorescent probes for nitric oxide and hydrogen peroxide in cell signaling. Curr. Opin. Chem. Biol. 11(6), 620-625 (2007). 
11 Lim MH, Xu D, Lippard SJ. Visualization of nitric oxide in living cells by a copper-based fluorescent probe. Nat. Chem. Biol. 2(7), 375-380 (2006).

Tsikas D. Methods of quantitative analysis of the nitric oxide metabolites nitrite and nitrate in human biological fluids. Free Radic. Res. 39(8), 797-815 (2005).

13 Gladwin MT, Schechter AN, Kim-Shapiro DB et al. The emerging biology of the nitrite anion. Nat. Chem. Biol. 1(6), 308-314 (2005).

14 Tsikas D. Analysis of nitrite and nitrate in biological fluids by assays based on the Griess reaction: appraisal of the Griess reaction in the $\mathrm{L}$-arginine/nitric oxide area of research. J. Chromatogr. B Analyt. Technol. Biomed. Life Sci. 851(1-2), 51-70 (2007).

15 Giustarini D, Rossi R, Milzani A, Dalle-Donne I. Nitrite and nitrate measurement by griess reagent in human plasma: evaluation of interferences and standardization. In: Methods in Enzymology. Enrique C, Lester P (Eds). Academic Press, CA, USA, 361-380 (2008).

16 Nussler AK, Bruckner UB, Vogt J, Radermacher P. Measuring end products of nitric oxide in vivo. In: Methods in Enzymology. Enrique C, Lester P (Eds). Academic Press, CA, USA, 75-83 (2002).

17 Schwarz A, Modun D, Heusser K et al. Stable-isotope dilution GC-MS approach for nitrite quantification in human whole blood, erythrocytes, and plasma using pentafluorobenzyl bromide derivatization: nitrite distribution in human blood. J. Chromatogr. B Analyt. Technol. Biomed. Life Sci. 879(17-18), 1485-1495 (2011).

18 Macarthur PH, Shiva S, Gladwin MT. Measurement of circulating nitrite and $S$-nitrosothiols by reductive chemiluminescence. J. Chromatogr. B Analyt. Technol. Biomed. Life Sci. 851(1-2), 93-105 (2007).

19 Dejam A, Hunter CJ, Pelletier MM et al. Erythrocytes are the major intravascular storage sites of nitrite in human blood. Blood 106(2), 734-739 (2005).

20 Basu S, Wang X, Gladwin MT, Kim-Shapiro DB. Chemiluminescent detection of S-nitrosated proteins: comparison of tri-iodide, copper/co/cysteine, and modified copper/cysteine methods. In: Methods in Enzymology, Enrique C, Lester P (Eds). Academic Press, CA, USA, 137-156 (2008).

21 Doctor A, Platt R, Sheram ML et al. Hemoglobin conformation couples erythrocyte $S$-nitrosothiol content to O-2 gradients. Proc. Natl. Acad. Sci. US A 102(16), 5709-5714 (2005).

22 Paulsen CE, Carroll KS. Cysteine-mediated redox signaling: chemistry, biology, and tools for discovery. Chem. Rev. 113(7), 4633-4679 (2013).

23 Wang H, Xian M. Chemical methods to detect $S$-nitrosation. Curr. Opin. Chem. Biol. 15(1), 32-37 (2011).

24 Bechtold E, King SB. Chemical methods for the direct detection and labeling of $S$-nitrosothiols. Antioxid. Redox. Signal. 17(7), 981-991 (2012).

25 Pan J, Downing JA, Mchale JL, Xian M. A fluorogenic dye activated by $S$-nitrosothiols. Mol. BioSyst. 5(9), 918-920 (2009).

26 Seneviratne U, Godoy LC, Wishnok JS, Wogan GN, Tannenbaum SR. Mechanism-based triarylphosphine-ester probes for capture of endogenous RSNOs. J. Am. Chem. Soc. 135(20), 7693-7704 (2013). 